\title{
One-way fluid structure interaction of pipe under flow with different boundary conditions
}

\author{
M. Dahmane*, D. Boutchicha*, L. Adjlout** \\ *LMA, Mechanical EngineeringDepartment, USTO-MB, BP 1055 El Menaour, Oran 31000, Algeria, \\ E-mail:dahmanemoul@gmail.com \\ **Mechanical Engineering Faculty, USTO-MB, BP 1055 El Menaour, Oran 31000, Algeria, E-mail: adjlout@yahoo.fr \\ cross $^{\text {ref }}$ http://dx.doi.org/10.5755/j01.mech.22.6.13189
}

\section{Introduction}

Hydraulic induced vibration starts with a continuous flow disturbance that creates a periodic pressure pulse. At changes in direction (elbow, tee, bend) or changes in flow cross section (valve, orifice, reducer) this pressure pulse causes pulsating forces on the pipe and makes it vibrate [1]. The complexity of the vibro-acoustic behaviour of flexible, fluid-filled pipe systems is predominantly determined by two parameters: the frequency and the ratio of the masses per unit pipe length of fluid and pipe wall. The number of simultaneously propagating waves in the pipes increases with frequency. The mass ratio determines whether fluid borne and structure-borne waves may be treated separately. If this ratio is close to one, fluid pulsations and mechanical vibrations will be strongly coupled, so that it becomes necessary to consider their interaction when analyzing the dynamic behavior [2]. Therefore, it is vitally important that the evaluation of natural frequency of a pipe be accurately to prevent any resonance condition. There have been extensive studies on the modeling and analysis of fluid conveying pipes over the past half-century, as reported by Paidoussis and al [3, 4], the pipe conveying fluid has established itself as a generic paradigm of a kaleidoscope of interesting dynamical behaviour. The effect of internal flow on transverse vibration of a pipe was studied by [3]. Coriolis acceleration of the internal fluid was taken into account in $[5,6]$.

In practice, the geometry of fluid conveying pipe systems is generally complicated. For these problems, analytical methods are not sufficient for the vibration analysis of real pipeline systems. Therefore, it is desirable to utilize numerical or approximately analytical methods such as finite element method (FEM) [7], (FEM)-state space approach [8], spectral element method (SEM) [9], transfer matrix method (TMM) [10], differential transformation method (DTM) [11], generalized integral transform technique (GITT) [12] and differential quadrature element method-(DQEM) proposed for obtaining highly accurate natural frequencies of multiple-stepped beams with an aligned neutral axis[13] can be mentioned. The problem of nonlinearities can be found in [14] by considering the dynamical behaviour of a fluid-conveying curved pipe subjected to motion-limiting constraints and a harmonic excitation. Based on a Newtonian method, the in-plane equation of motion of this curved pipe is derived. Then a set of discrete equations in spatial space obtained by the differential quadrature method (DQM) is solved numerically. In his study, H.R. ÖZ [15] considered the non-linear transverse vibrations of highly tensioned pipes conveying fluid as being investigated.

Practically, long, cross-country pipelines rest on an elastic medium such as a soil, and hence, a model of the soil medium must be included in the analysis. Chellapilla and al [16], studied the effect of a Pasternak foundation on the critical velocity of a fluid-conveying pipe. Chellapilla and al [17] studied problem of vibrations of fluid-conveying pipes resting on a two-parameter foundation model such as the Pasternak-Winkler model. This work has been extended to the study of the effect of the Pasternak foundation on the natural frequencies of the pipeline for the pinned-pinned, clamped-clamped and clamped-pinned boundary conditions. It is well established from published literature where there exists a critical velocity of the fluid near which the natural frequency of the pipeline tends to zero. Dynamic response of linear systems subjected to dynamic loading, such as shock or seism, with finite element procedures is of paramount importance in many engineering applications [18]. Advances in finite element methodology have made it possible to simulate the dynamic motion of the fluid coupled with the flexible pipe for arbitrary geometries within the context of a general purpose finite element program [19].

Numerical enhancement of the finite element code ANSYS and in order to produce coupled fluid-structure dynamic analysis with pressure-based formulation has been exposed and validated [20], using modal and spectral methods. Enhancement of the modeling possibilities within the ANSYS code is carried out with implementation of fluidstructure symmetric formulations for elasto-acoustic and hydro-elastic problems. Using symmetric formulation enables linear dynamic analysis with modal projection techniques for a fluid-structure coupled system. In the mentioned study, comparison of numerical results has been performed for modal analysis for (3D) using modal methods (temporal and spectral approaches). Taking fluid-structure interaction into account in such problems is made possible by finite element analysis (FEA), using ANSYS code [19]. This latter paper presents a state-variable model developed for the analysis of fluid-induced vibration of composite pipeline systems. Simply supported, clamped and clampedsimply supported pipelines are investigated. The influence of fluids Poisson ratio, the ratio of pipe radius to pipe-wall thickness, the ratio of liquid mass density to pipe-wall mass density, the fluid velocity, initial tension and fluid pressure are all considered. The effected numerical simulations to rest most of the time on a modeling simplified of one of the two mediums, or appeal specified procedures of coupling which permit to function together the computer codes specially, is developed for fluid from on a side and the structure of the other [21]. 
In this work, the fluid is considered incompressible and the solid is an elastic body. Therefore, this study will be devided into two parts. The first part is specified for analytical modeling and formulas of natural frequencies and shapes modes, by establishing the equations of motions of the pipe with different boundary conditions. One may consider a two-mode Galerkin approximation has been employed to get results for the pinned-pinned beam. Only the effect of fluid flow was taken into account, and the theoretical eigen-frequencies are obtained, under different fluid velocities. For the numerical approach, the interaction enters the fluid and the solid involves the velocity continuity and the mechanical equilibrium condition to the interface. This interaction is based on a procedure of instantaneous coupling, where they obtained pressure in iterations of fluid numerical model is used as extern solicitation for the solid numerical model. Unidirectional approach has been used for the three cases; pinned-pinned, clamped-pinned and clamped-clamped.

\section{The analytical model}

\subsection{Equations of motion and boundary conditions}

The pipe is long and straight, thus facilitating the use of Euler-Bernoulli beam theory; the motions are small so that the system can be analyzed by the linear theory; and, the effects of internal pressure and external forces are neglected in the analysis [3]. By applying Newton's second law of motion for afluid-conveying pipe of length $\mathrm{L}$ with lateral displacement $w$, four coupled, linear pipe-dynamic equations have been derived in the previous work [4] as follow:

$$
\begin{aligned}
& E I \frac{\partial^{4} w}{\partial x^{4}}(x, t)+m_{a} V_{0}^{2} \frac{\partial^{2} w}{\partial x^{2}}(x, t)+2 m_{a} V_{0} \frac{\partial^{2} w}{\partial x \partial t}(x, t)+ \\
& +\left(m_{a}+m_{s}\right) \frac{\partial^{2} w}{\partial t^{2}}(x, t)=0 ;
\end{aligned}
$$

a) Pinned-Pinned Pipe:

$$
x=0 \rightarrow\left\{\begin{array}{c}
w(0, t)=0 ; \\
w^{\prime \prime}(0, t)=0 ;
\end{array} \quad x=L \rightarrow\left\{\begin{array}{c}
w(L, t)=0 \\
w^{\prime \prime}(L, t)=0
\end{array}\right.\right.
$$

b) Clamped-Pinned Pipe:

$$
x=0 \rightarrow\left\{\begin{array}{c}
w(0, t)=0 ; \\
w^{\prime}(0, t)=0 ;
\end{array} \quad x=L \rightarrow\left\{\begin{array}{c}
w(L, t)=0 ; \\
w^{\prime \prime}(L, t)=0 .
\end{array}\right.\right.
$$

c) Clamped-Clamped Pipe:

And those for a clamped-clamped pipe are:

$$
x=0 \rightarrow\left\{\begin{array}{c}
w(0, t)=0 ; \\
w^{\prime}(0, t)=0 ;
\end{array} \quad x=L \rightarrow\left\{\begin{array}{c}
w(L, t)=0 \\
w^{\prime}(L, t)=0
\end{array}\right.\right.
$$

The dimensionless form of Eq. (1) can be written as:

$$
\eta^{\prime \prime \prime \prime}+u^{2} \eta^{\prime \prime}+2 \beta^{1 / 2} u \dot{\eta}^{\prime}+\ddot{\eta}=0
$$

where

$$
\left.\begin{array}{l}
(\dot{\eta})=\partial(\eta) / \partial \tau \text { and }(\eta)^{\prime}=\partial(\eta) / \partial \xi ; \\
\xi=\frac{x}{L} ; \eta=\frac{w}{L} ; \tau=\left[\frac{E I}{m_{a}+m_{s}}\right]^{1 / 2} \frac{1}{L^{2}} t ; \\
\beta=\frac{m_{s}}{m_{a}+m_{s}} ; u=\left[\frac{m_{a}}{E I}\right]^{1 / 2} V_{0} L .
\end{array}\right\}
$$

According to Galerkin's method, the solution of Eq. (1) may be expressed as:

$$
w(x, t)=\mathfrak{R} e\left[\emptyset_{r}\left(\frac{x}{L}\right) e^{i \omega t}\right],
$$

where, $\Re e$ denotes the real part, $\omega$ denotes the mode of vibration and where $\emptyset_{r}(\xi)$ are the dimensionless eigen-functions of a beam with the same boundary conditions as the pipe under consideration, $\varnothing_{r}(\xi)$ given by [22]:

$$
\emptyset_{r}(\xi)=C_{r}\left[\left(\cos \alpha_{r} \xi-\operatorname{ch} \alpha_{r} \xi\right)+\sigma_{r}\left(\sin \alpha_{r} \xi-\operatorname{sh} \alpha_{r} \xi\right)\right]
$$

where the constants $C_{r}, r=1$ to 4 are determined into the boundary conditions; the frequency functions $\sigma_{r}=\left(\frac{\operatorname{ch} \alpha_{r} L-\cos \alpha_{r} L}{\operatorname{sh} \alpha_{r} L-\sin \alpha_{r} L}\right)$.

In the above equation, $\alpha_{r}$ is the frequency parameter of the pipe without fluid flow, which is considered as a beam, and it's values are:

- $\alpha_{r} L=n \pi ; r=1,2,3 \ldots N$ for the pinnedpinned case;

$$
\text { - } \alpha_{1} L=3.9266 ; \alpha_{2} L=7.0685 ; \alpha_{3} L=10.2101
$$

for the pinned-clamped case;

- $\alpha_{1} L=4.7300 ; \alpha_{2} L=7.8532 ; \alpha_{3} L=10.9956$ for the clamped -clamped case.

The equation (8) is substituted into the left-hand side of Eq. (5), the result will generally not be zero, but equal to an error function, which may be denoted by $\mathrm{\epsilon}_{N}\left[w_{N}(x, t)\right]$. Galerkin's method requires that:

$$
\int_{0}^{1} \epsilon_{N}\left[w_{N}(x, t)\right] \emptyset_{r}\left(\frac{x}{L}\right) d x=0 .
$$

Substituting Eq. (9), we obtain:

$$
\epsilon_{N}\left(\varnothing_{r}\right)=\sum_{1}^{\infty} a_{r}\left(\begin{array}{l}
E I \emptyset_{r}^{\prime \prime \prime}+m_{a} V_{0}^{2} \emptyset_{r}^{\prime \prime}+2 i m_{a} V_{0} \omega \emptyset_{r}^{\prime}- \\
-\left(m_{a}+m_{s}\right) \omega^{2} \emptyset_{r}
\end{array}\right) .
$$

Using the non-dimensional parameters, we obtain:

$$
\epsilon_{N}\left(\emptyset_{r}\right)=\sum_{1}^{\infty} a_{r}\left(\emptyset_{r}^{\prime \prime \prime}+u^{2} \emptyset_{r}^{\prime \prime}+2 i \beta^{1 / 2} u \Omega \emptyset_{r}^{\prime}-\Omega^{2} \emptyset_{r}\right)
$$




$$
\Omega=\omega L^{2}\left(\frac{m_{a}+m_{s}}{E I}\right)^{1 / 2} .
$$

The dimensionless

frequency $\Omega=\mathfrak{R e}(\Omega)+i \Im m(\Omega)$ is plotted parametrically with the dimensionless flow velocity $u$ [23], where $\mathfrak{R e}(\Omega)$ the real part and $\mathfrak{I} m(\Omega)$ the imaginary part.

$$
\mathfrak{R} e(\Omega)>\mathfrak{I} m(\Omega) .
$$

Multiplying Eq. (8) by $\varnothing_{s}$ and using the orthogonal property of the eigen-functions and integration over the domain $[0,1]$ yields:

$$
\left.\begin{array}{l}
a_{r}\left(\alpha_{r}^{4}-\Omega^{2}\right)+u^{2} \sum_{s=1}^{\infty}\left(a_{r} C_{s r}\right)+2 i \beta^{1 / 2} u \Omega \sum_{s=1}^{\infty}\left(a_{r} b_{s r}\right)=0 \\
s=1,2 \ldots N,
\end{array}\right\}
$$

where the different matrices are defined as follows:

$$
b_{s r}=\int_{0}^{1} \psi_{s} \psi_{r}^{\prime} d \xi ; \quad C_{s r}=\int_{0}^{1} \psi_{s} \psi_{r}^{\prime \prime} d \xi .
$$

lows:

The Eq. (15) may be written in matrix form as fol-

First, one may consider a two-mode Galerkin approximation of the system, namely:

$$
\left(\left[\begin{array}{cc}
-\Omega_{j}^{2} & 0 \\
0 & -\Omega_{j}^{2}
\end{array}\right]\left[\begin{array}{cc}
2 i \beta^{1 / 2} u \Omega_{j} b_{s r} & -2 i \beta^{1 / 2} u \Omega_{j} b_{s r} \\
2 i \beta^{1 / 2} u \Omega_{j} b_{s r} & 2 i \beta^{1 / 2} u \Omega_{j} b_{s r}
\end{array}\right]\left[\begin{array}{cc}
\alpha_{r}^{4}+u^{2} C_{s r} & u^{2} C_{s r} \\
u^{2} C_{s r} & \alpha_{r}^{4}+u^{2} C_{s r}
\end{array}\right]\right)\{a\}=0 .
$$

For pinned ends $b_{r r}=0, b_{s r}=-b_{r s}$ and $C_{r s}=0$. Eq. (17) may be written as:

$$
\left(\left[\begin{array}{cc}
-\Omega_{j}^{2} & 0 \\
0 & -\Omega_{j}^{2}
\end{array}\right]\left[\begin{array}{cc}
0 & -2 i \beta^{1 / 2} u \Omega_{j} b_{s r} \\
2 i \beta^{1 / 2} u \Omega_{j} b_{s r} & 0
\end{array}\right]\left[\begin{array}{cc}
\alpha_{r}^{4}+u^{2} C_{s r} & 0 \\
0 & \alpha_{r}^{4}+u^{2} C_{s r}
\end{array}\right]\right)\{a\}=0 .
$$

Eq. (16) may be written in matrix form as follows:

$$
\left[K-\Omega_{j}^{2} M I\right]\{a\}=0,
$$

where $K$ is the stiffness matrix, $I$ is the identity matrix and $a^{T}=\left\{a_{1}, a_{2}, \ldots a_{n}\right\}$.

Setting the determinant ofthe coefficient matrix above equal to zero and retaining thefirst two terms of the above equation, we get the frequency equation. For the pinned -pinned case it is:

$$
\begin{gathered}
\Omega_{j}^{4}-\left[\lambda_{1}^{4}+\lambda_{2}^{4}+\left(C_{11}+C_{22}+4 \beta b_{12}^{2}\right) u^{2}+\right] \Omega_{j}^{2}+ \\
+\left[\lambda_{1}^{4} \lambda_{2}^{4}+u^{2}\left(\lambda_{2}^{4} C_{11}+\lambda_{1}^{4} C_{22}\right)+u^{4} C_{11} C_{22}\right]=0 ; \\
\Omega_{j}=0 \Rightarrow \lambda_{1}^{4} \lambda_{2}^{4}+u^{2}\left(\lambda_{2}^{4} C_{11}+\lambda_{1}^{4} C_{22}\right)+u^{4} C_{11} C_{22}=0 .
\end{gathered}
$$

The critical velocity parameter for the mode 1 : $u_{c r}=N \pi$.

Table 1

Integral values $b_{s r}, b_{r r}, C_{s r}, C_{r r}$ for pinned-pinned pipe by [4]

\begin{tabular}{|c|c|}
\hline Parameters & Pinned-pinned pipes \\
\hline$b_{s r}$ & $\frac{2 \alpha_{r} \alpha_{s}}{\left(\alpha_{r}^{2}-\alpha_{s}^{2}\right)}\left[(-1)^{s+r}-1\right]$ \\
\hline$b_{r r}$ & 0 \\
\hline$C_{s r}(s \neq r)$ & 0 \\
\hline$C_{r r}$ & $-\alpha_{r}{ }^{2}$ \\
\hline
\end{tabular}

\subsection{Interpretation and analytical results}

The frequencies parameters for the first two natural modes are calculated bending movement of a pipe in different boundary conditions. The results are presented for the following cases: 1) Fluid conveying pipe without foundation; 2) No-flow, pipe with parameter elastic foundation and 3) Fluid conveying pipe with Winkler foundation. Comparison has been made with available literature wherever possible. For the pinned-pinned boundary condition, numerical results have been obtainedconsidering the first two terms of the equation resulting from using Galerkin approximation.

Case 1: Fluid conveying pipe without foundation

Results have been obtained for the pipe with fluid (no elastic foundation). They are compared with available literature. Table 2 show the two first frequencies parameter for pinned-pinned fluid-conveying pipe. The two methods employed for comparison are Fourier series and Galerkin.

The error on the frequencies of the order 0.075 is very satisfactory. The Fig. 1 watches the effect Coriolis on the vibration of a pinned-pinned pipe with flow.

Case 2: Pipe with elastic foundation (Winkler model) for no flow

For the pinned-pinned fluid-conveying pipes $(\beta \neq 0)$, however, the effect of an elastic foundation is stabilizing, as shown by [4]. Galerkin expansion was employed to calculate numerical solutions, for the Pasternak-Winkler model [16].

The terms $k_{1} w$ and $k_{2} \frac{\partial^{2} w}{\partial x^{2}}$ are added to the equation of motion which becomes as the following: 


$$
\begin{aligned}
& E I \frac{\partial^{4} w}{\partial x^{4}}(x, t)+\left(m_{a} V_{0}^{2}-k_{2}\right) \frac{\partial^{2} w}{\partial x^{2}}(x, t)+k_{1} w+\quad \begin{array}{c}
\text { Non-dimensional frequency equation for the } \\
\text { pinned-pinned pipes with elastic foundation is: }
\end{array} \\
& +2 m_{a} V_{0} \frac{\partial^{2} w}{\partial x \partial t}(x, t)+\left(m_{a}+m_{s}\right) \frac{\partial^{2} w}{\partial t^{2}}(x, t)=0 . \\
& \Omega_{j}^{4}-\left[\begin{array}{l}
\lambda_{1}^{4}+\lambda_{2}^{4}+\left(C_{11}+C_{22}\right)\left(u^{2}-\gamma_{2}\right)+ \\
+4 \beta b_{12}^{2}\left(u^{2}-\gamma_{2}\right)+2 \gamma_{1}
\end{array}\right] \Omega_{j}^{2}+\left[\begin{array}{l}
\left(\lambda_{1}^{4}+\gamma_{1}\right)\left(\lambda_{2}^{4}+\gamma_{1}\right)+\left(u^{2}-\gamma_{2}\right)\left\{\left(\lambda_{2}^{4}+\gamma_{1}\right) C_{11}+\left(\lambda_{1}^{4}+\gamma_{1}\right) C_{22}\right\}+ \\
+\left(u^{2}-\gamma_{2}\right)^{2}\left(C_{11} C_{22}\right)
\end{array}\right]=0 .
\end{aligned}
$$

The two first frequencies parameter pinned-pinned fluid-conveying pipe, comparison Fourier series and Galerkin method for various values of $u$

\begin{tabular}{|c|c|c|c|c|c|c|}
\hline$u$ & $\Omega_{1}$ Fourier series & $\Omega_{1}$ Galerkin method & $\%$ Var. & $\Omega_{2}$ Fourier series & $\Omega_{2}$ Galerkin method & $\%$ Var. \\
\hline 0.0 & 9.869 & 9.8685 & 0.005 & 39.478 & 39.4635 & 0.036 \\
\hline 0.1 & 9.864 & 9.8603 & 0.037 & 39.475 & 39.4604 & 0.037 \\
\hline 0.3 & 9.820 & 9.8163 & 0.037 & 39.466 & 39.4362 & 0.075 \\
\hline 0.5 & 9.731 & 9.7281 & 0.029 & 39.401 & 39.3863 & 0.037 \\
\hline 1.0 & 9.310 & 9.3046 & 0.054 & 39.168 & 39.1532 & 0.037 \\
\hline 1.5 & 8.574 & 8.5705 & 0.035 & 38.773 & 38.7583 & 0.037 \\
\hline 2.0 & 7.455 & 7.4515 & 0.035 & 38.208 & 38.1932 & 0.037 \\
\hline 2.5 & 5.779 & 5.7751 & 0.039 & 37.456 & 37.4417 & 0.036 \\
\hline 3.0 & 2.784 & 2.7790 & 0.050 & 36.497 & 36.4822 & 0.037 \\
\hline 3.141 & 0.0 & 0.0 & 0.0 & 36.183 & 36.1699 & 0.033 \\
\hline
\end{tabular}

$$
\% \text { Variation }=\left|\frac{\Omega_{\text {Galerkin method }}-\Omega_{\text {Fourier series }}}{\Omega_{\text {Fourier series }}}\right| \times 100
$$

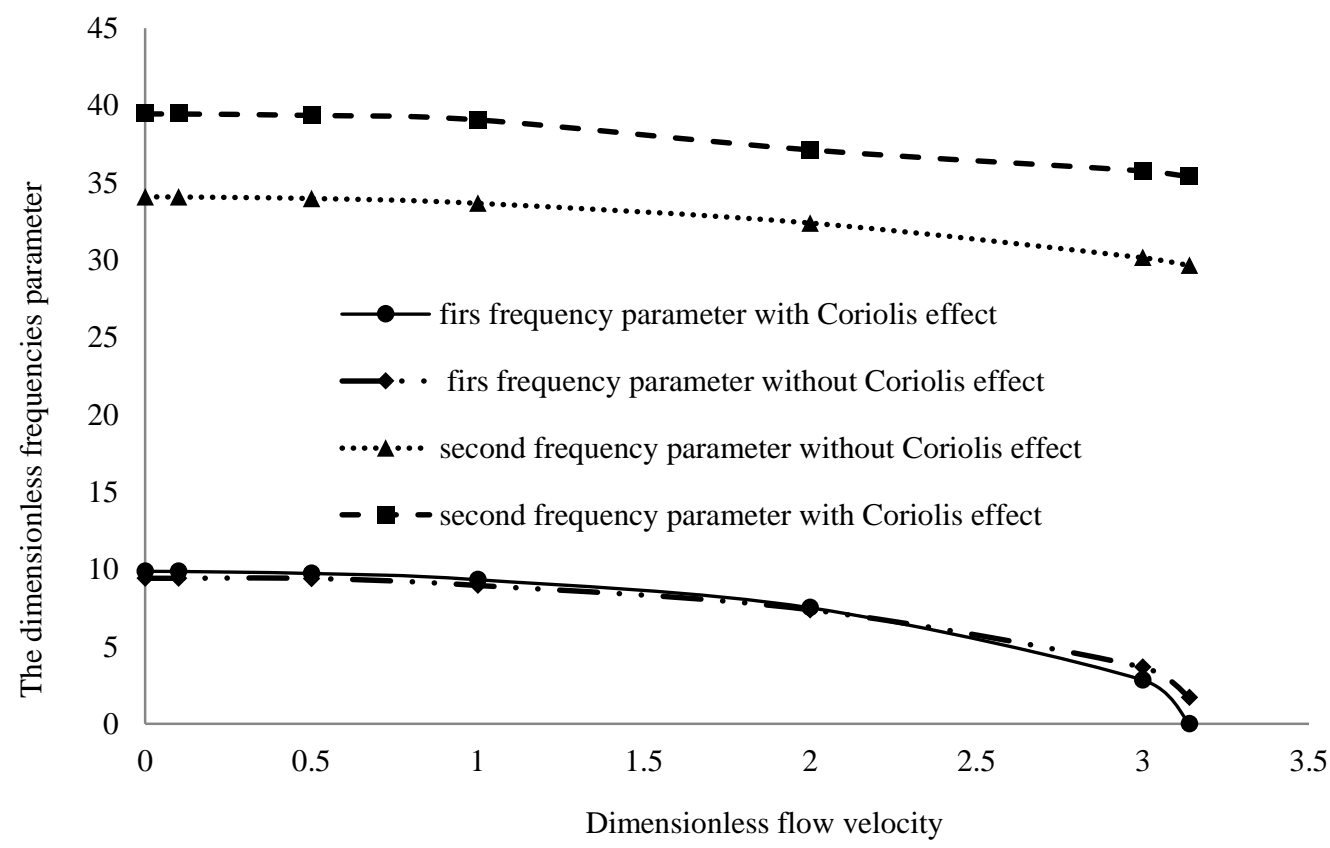

Fig. 1 The effect Coriolis on the vibration of a pinned-pinned pipe with flow

Results for no-flow have been presented for parameter elastic foundation (the presence of the Winkler foundation) and compared with available literature. Table 3 shows the values of the first frequency parameter for a pinnedpinned pipe for different values of $\gamma_{1}$. There is very strongly an increase for $\gamma_{1}$ in the surrounding by $10^{4}$.

Case 3: Fluid-conveying pipe with elastic foundation (Winkler model)
Results for a pipe with fluid flow have been presented for various values of $\gamma_{1}$ (Winkler foundation), $u$ and $\beta$ is 0.1 . According to the Table 4 case of a pinned-pinned pipe, one notice that the first eigen-frequency increases appreciably with the values of the $\gamma_{1}$, very strongly increases for $\gamma_{1}$ in the surrounding by $10^{3}$. According to Table 4 , the Winkler foundation has a stabilizing effect in the pipe and increasing values of $\gamma_{1}$ tend to increase both the critical flow velocity and the first frequency parameter pinned-pinned fluid-conveying pipe. 
The first frequency parameter pinned-pinned pipe for various values $\gamma_{1}$

\begin{tabular}{|c|c|c|c|}
\hline$\gamma_{1}$ & $\Omega_{1}($ Ref.[17] $)$ & $\Omega_{1}$ present & $\%$ Variation \\
\hline 0.0 & 9.865 & 9.869 & 0.040 \\
\hline $10^{2}$ & 14.047 & 14.049 & 0.014 \\
\hline $10^{4}$ & 100.480 & 100.491 & 0.010 \\
\hline
\end{tabular}

$$
\% \text { Variation }=\left|\frac{\Omega_{1} \text { present }-\Omega_{1}(\operatorname{Ref} .[17])}{\Omega_{1}(\operatorname{Ref} .[17])}\right| \times 100
$$

Table 4

The first frequency parameter pinned-pinned fluid-conveying pipe for various values of $\gamma_{1}, u$ and $\beta=0.1$

\begin{tabular}{|c|c|c|c|c|c|}
\hline \multicolumn{7}{|c|}{$\gamma_{1}$} \\
\hline$u$ & 0.01 & 0.5 & 2.5 & 10 & 100 \\
\hline 0.0 & 9.8695 & 9.8943 & 9.9949 & 10.3632 & 14.0498 \\
\hline 1.0 & 9.3463 & 9.3730 & 9.4789 & 9.8484 & 13.4649 \\
\hline 2.0 & 7.5792 & 7.6112 & 7.7404 & 8.1175 & 12.5138 \\
\hline 3.1415 & 0.0988 & 0.6987 & 1.5624 & 2.3882 & 9.8822 \\
\hline 3.1416 & 0.0 & 0.2677 & 1.2134 & 2.3834 & 9.8801 \\
\hline 3.1495 &. & 0.0 & 0.6331 & 2.2785 & 9.8580 \\
\hline 3.1953 &. &. & 0.0 & 2.3834 & 9.7107 \\
\hline 3.2988 &. &. &. & 0.0 & 9.3521 \\
\hline 4.4723 &. &. &. &. & 0.0 \\
\hline
\end{tabular}

\section{The numerical model}

3.1. Fluid-structure interaction problems in the ANSYS code

Design of structures in contact with fluid needs specific attentions on the static and dynamic analysis and requires more accurate simulation tools (finite element method) to improve performance of the system while minimizing the cost of manufacturing. The commercial package ANSYS was used with Fluent for computational fluid dynamics and ANSYS mechanical for the structural simulation. The traditional approach to determine the mechanical stress and deflection of a structure in contact with fluid consists of a steady state computational fluid dynamics (CFD) simulation which provides the fluid pressure on the structure. This can then be applied as a boundary or load condition for the FEM simulation of the configuration, like in Schmucker and al (Ref. [24]).

Fluid Model

- The simulation has been realized under the following conditions:

- The fluid is Newtonian and incompressible, Flow 3D is turbulent.

- Mesh with hexahedral elements, (hexahedral) 8 nodes.

\section{Structure Model}

The object of this section is the determination of proper frequencies of the right pipe, using a tridimensional element and the study of proper from for each case.

The conditions: the length;
- Elastic modulus of material component the structure is chose $2.0 * 10^{11}\left[\mathrm{~N} / \mathrm{m}^{2}\right]$, while the solid density, the Poisson's ratio are accepted respectively $7850\left[\mathrm{Kg} / \mathrm{m}^{3}\right]$, 0.26 ;

- The used element for the simulation (mesh), from the ANSYS Mechanical User Guide is the hexahedral (three-dimensional) known under «SOLID 45», using eight nodes isotropic.

\subsection{Fluid-Structure coupling conditions}

ANSYS Workbench permits of making a simulation FSI (Fluid Structure Interaction) in a very easier manner, the schema the charged transfer, which is in our case the pressure.

The coupling conditions consist of transfer fieldfluid operation on the structure and concern the pressure transfer on solid. The types of transfer are the following:

1.The fluid cod transmit the pressure, interface nodal, on structure code

2. The structure code calculates the solution and put on again nodes coordinates.

3. The structure transmits new position of interface nodes. In (Workbench) the simulation is done with Mechanical code, see Fig. 3.

\subsection{Interpretation and numerical results}

The results are presented for the following cases: 1) Fluid-conveying pipe without foundation; 2) No-flow, pipe with parameter elastic foundation and 3) Fluid conveying pipe with Winkler foundation. Numerical results have been obtained considering the first two frequencies. To validate the convergence between the analytical method and the numerical one, a typical uniform model is used according to the length of profiles. For this, we considered the profile of the pipe as a model: pinned-pinned beam, pinnedclamped and clamped-clamped beam of a uniform circular section. The commercial finite element program ANSYS is employed for modeling the steel pipeline using eight node isotropic 3D solid elements (SOLID 45). Fig. 2 shows the profile tube mesh with hexahedral elements. Fig. 3 shows fluid-structure coupling is only modeled between the elastic structure and the fluid (the hydrodynamic load is imported) of clamped-clamped pipe for mode 1. In the ANSYS code, this is achieved with 45390 elements, (hexahedral) fluid finite elements (3D element with eight nodes and three degrees of freedom). Fig. 4 shows the plot of the displacements nodal of clamped-clamped pipe for mode 1 .

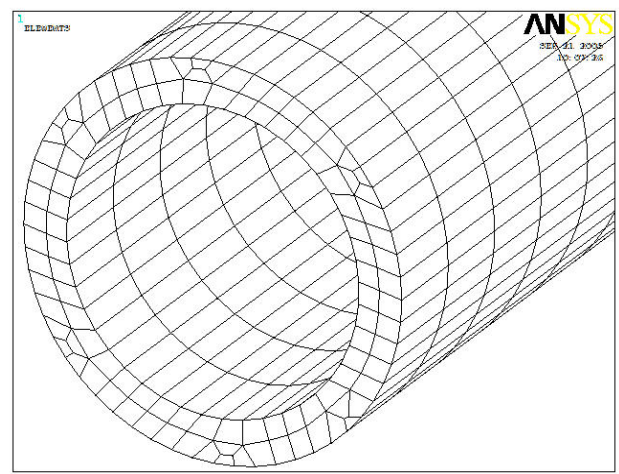

Fig. 2 The profile tube mesh with hexahedral elements (SOLID 45) 


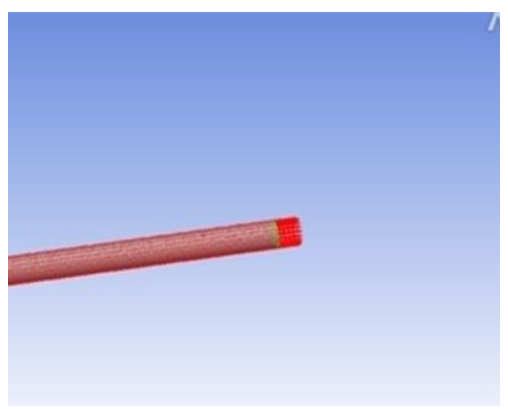

Fig. 3 Represents the distribution of the constraints of pressure on the level of the interface of clamped-clamped pipe (mode 1): imported pressure

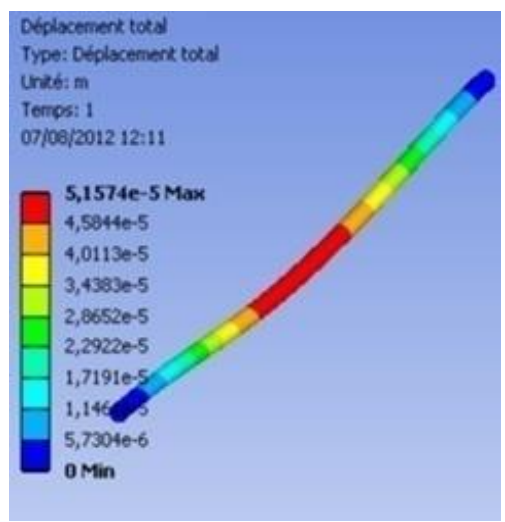

Fig. 4 Represents the distribution of the displacements of clamped-clamped pipe (mode 1 )
Case 1: Fluid conveying pipe without foundation

The first two vibration frequencies as functions of fluid velocities in the simply-supported, clamped and clamped-simply supported pipes without foundation were determined and are shown in Table 5 and Figs. 5-6, respectively. In these table and figures, values of the velocity $V_{0}$ are varying from zero to the value $V_{0}=103.17 \mathrm{~m} / \mathrm{s}$ which corresponds of the velocity parameter $u=2$ for the mass ratios parameter $\beta$ is 0.6 . For boundary conditions pinnedpinned, we notice a good agreement with the results presented analytically for this case, the variation on the first frequency of the order 0.80 is very satisfactory.

Case 2: Pipe with elastic foundation (Winkler model) for no flow

The first two vibration frequencies as a function of elastic foundation (Winkler model) for no flow where

$V_{0}=0 \mathrm{~m} / \mathrm{s}$ (where the velocity parameter $u=0$ ) and $\beta=0.5$ in the simply-supported and clamped-simply supported pipes were determined and are shown in Tables 6 and 7. A similar trend is noticed in these cases, the error on the first frequency of the order 1.15 is very satisfactor.

Case 3: Fluid-conveying pipe with elastic foundation (Winkler model)

The first two vibration frequencies as a function of fluid velocities in the clamped-clamped pipe with elastic foundation were determined and are shown in tables. 8 and 10 , respectively. For this case, values of the velocity $V_{0}$ are varying from zero to the value $103.17 \mathrm{~m} / \mathrm{s} \quad(u=2)$ for $\beta=0.6$.

Table 5

Two first natural frequencies (in Hz) pinned-pinned fluid-conveying pipe for various values of $u, \beta=0.6$

\begin{tabular}{|c|c|c|c|c|c|c|c|c|}
\hline \multirow[t]{2}{*}{$u$} & \multirow[t]{2}{*}{$V_{0}$} & \multicolumn{4}{|c|}{ Analytical results } & \multicolumn{2}{|c|}{ Numerical results } & \multirow{2}{*}{$\%$ Variation $f_{1}$} \\
\hline & & $\Omega_{1}$ & $\Omega_{2}$ & $f_{1}, \mathrm{~Hz}$ & $f_{2}, \mathrm{~Hz}$ & $f_{1}, \mathrm{~Hz}$ & $f_{2}, \mathrm{~Hz}$ & \\
\hline 0.00 & 0.00 & 9.8596 & 39.4780 & 58.5150 & 234.0596 & 58.8430 & 234.2900 & 0.56 \\
\hline 0.10 & 5.15 & 9.8466 & 39.4699 & 58.4379 & 234.2472 & 58.8410 & 234.3120 & 0.68 \\
\hline 0.20 & 10.31 & 9.8453 & 39.4647 & 58.4302 & 234.2163 & 58.4210 & 234.3100 & 0.18 \\
\hline 0.30 & 15.47 & 9.8240 & 39.4383 & 58.3037 & 234.0596 & 58.2470 & 234.3100 & 0.56 \\
\hline 0.40 & 20.63 & 9.7869 & 39.4383 & 58.0812 & 233.7036 & 58.2330 & 234.2660 & 0.26 \\
\hline 0.50 & 25.79 & 9.7402 & 39.3780 & 57.8064 & 233.7018 & 57.3400 & 234.1260 & 0.80 \\
\hline 1.00 & 51.58 & 9.2983 & 39.3119 & 55.1838 & 233.3095 & 55.4210 & 230.1320 & 0.42 \\
\hline 1.50 & 77.37 & 8.5753 & 38.7731 & 55.1838 & 230.1118 & 55.2835 & 230.0115 & 0.18 \\
\hline 2.00 & 103.17 & 7.4449 & 38.1691 & 44.1842 & 226.5272 & 44.3412 & 227.1233 & 0.35 \\
\hline
\end{tabular}

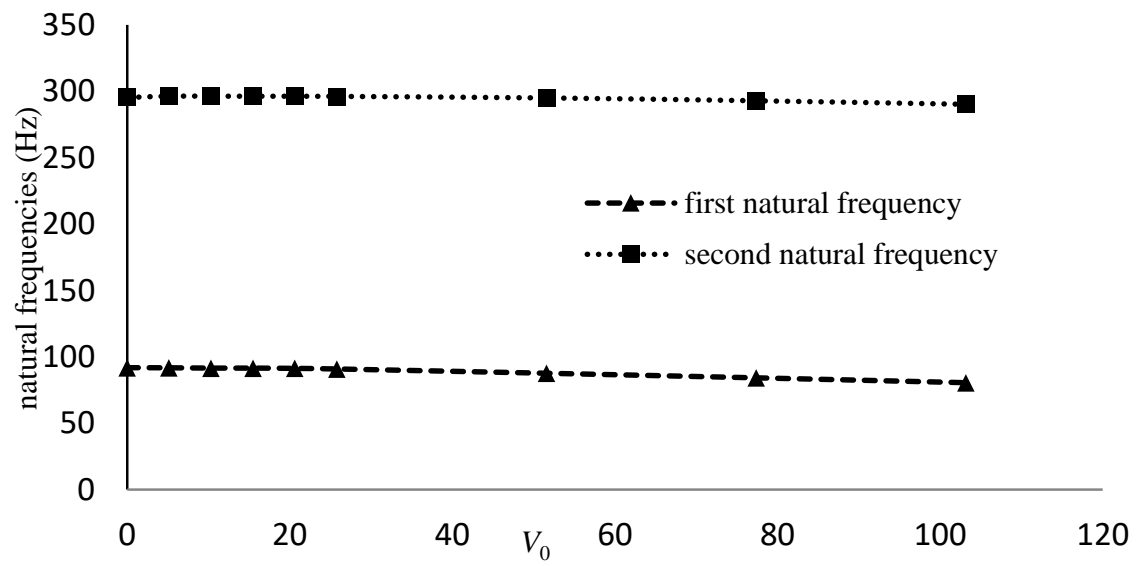

Fig. 5 Two first natural frequencies clamped-pinned pipe for various values of $V_{0}, \beta=0.6$ 


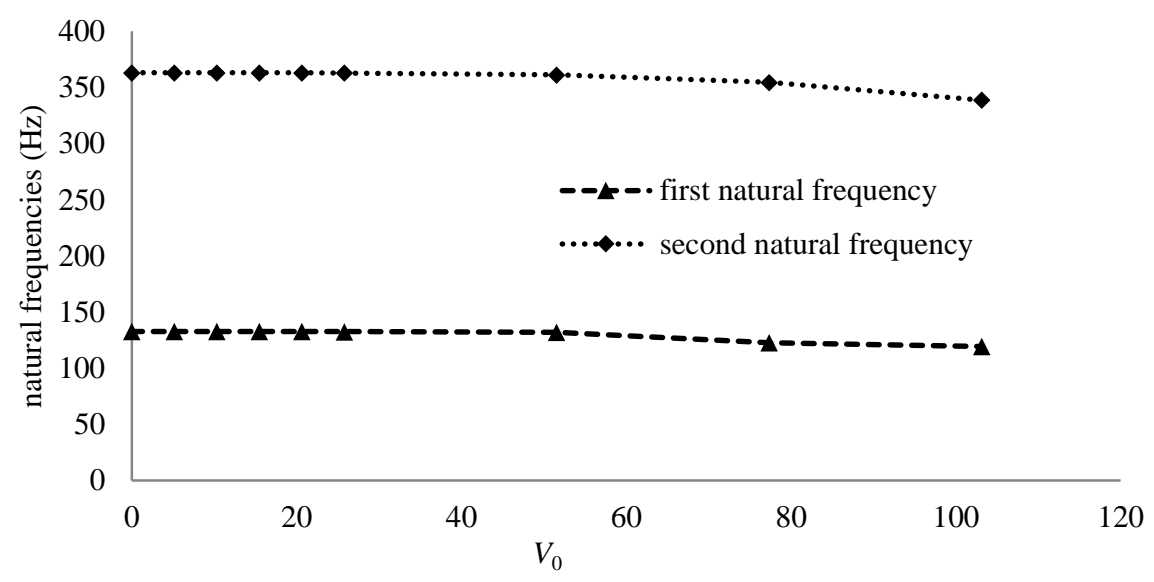

Fig. 6 Two first natural frequencies clamped-clamped pipe for various values of $V_{0}, \beta=0.6$

Table 6

Table 7

Two first natural frequencies (in $\mathrm{Hz}$ ) fluid-conveying pipe for various values of $\gamma_{1}$ where $\beta=0.5$

\begin{tabular}{|c|c|c|c|c|c|}
\hline \multirow{2}{*}{$\gamma_{1}$} & \multicolumn{4}{|c|}{ Pinned-pinned pipe } \\
\cline { 2 - 5 } & \multicolumn{2}{|c|}{$\begin{array}{c}\text { Numerical } \\
\text { results }\end{array}$} & \multicolumn{2}{c|}{$\begin{array}{c}\text { Analytical } \\
\text { results }\end{array}$} & \multirow{2}{*}{ \% Var. $f_{1}$} \\
\cline { 2 - 5 } & $f_{1}$ & $f_{2}$ & $f_{1}$ & $f_{2}$ & \\
\hline 0.01 & 58.84 & 234.79 & 58.57 & 234.09 & 0.45 \\
\hline 0.50 & 58.85 & 234.84 & 58.65 & 234.14 & 0.33 \\
\hline 2.50 & 59.56 & 235.01 & 58.87 & 234.91 & 1.15 \\
\hline 10 & 62.14 & 235.31 & 62.11 & 235.07 & 0.04 \\
\hline 100 & 78.36 & 241.51 & 78.13 & 241.22 & 0.29 \\
\hline 1000 & 212.56 & 309.53 & 211.96 & 308.93 & 0.28 \\
\hline 10000 & 231.56 & 577.25 & 231.12 & 576.85 & 0.19 \\
\hline
\end{tabular}

Two first natural frequencies (in $\mathrm{Hz}$ ) clamped-pinned fluid-conveying pipe for various values of $\gamma_{1}, \beta=0.5$

\begin{tabular}{|c|c|c|c|c|c|}
\hline \multirow{2}{*}{$\gamma_{1}$} & \multicolumn{5}{|c|}{ Clamped-pinned pipe } \\
\cline { 2 - 5 } & \multicolumn{2}{|c|}{ Numerical Results } & \multicolumn{2}{|c|}{$\begin{array}{c}\text { Analytical } \\
\text { Results }\end{array}$} & \multirow{2}{*}{ \% Var. $f_{1}$} \\
\cline { 2 - 5 } & $f_{1}$ & $f_{2}$ & $f_{1}$ & $f_{2}$ & \\
\hline 0.01 & 91.74 & 295.63 & 91.50 & 295.36 & 0.26 \\
\hline 0.50 & 91.78 & 295.66 & 91.54 & 295.39 & 0.26 \\
\hline 2.50 & 92.24 & 295.70 & 91.84 & 295.66 & 0.43 \\
\hline 10 & 93.68 & 296.34 & 93.08 & 296.05 & 0.64 \\
\hline 100 & 112.02 & 300.64 & 111.92 & 300.22 & 0.08 \\
\hline 1000 & 224.13 & 357.64 & 223.97 & 357.52 & 0.07 \\
\hline 10000 & 292.02 & 651.12 & 291.88 & 651.01 & 0.04 \\
\hline
\end{tabular}

$$
\% \text { Variation } f_{1}=\left|\frac{f_{1(\text { Numerical })-} f_{1(\text { Analytical })}}{f_{1(\text { Analytical })}}\right| \times 100
$$

Table 8

First natural frequencies (in $\mathrm{Hz}$ ) clamped-clamped fluid-conveying pipe for various values of $\gamma_{1}$ and $V_{0}, \beta=0$

\begin{tabular}{|c|c|c|c|c|c|c|}
\hline \multirow{2}{*}{$V_{0}, \mathrm{~m} / \mathrm{s}$} & \multicolumn{7}{|c|}{$\gamma_{1}$} \\
\cline { 2 - 7 } & 0.01 & 0.5 & 2.5 & 10 & 100 & 1000 \\
\hline 0.00 & 132.78 & 132.80 & 132.90 & 133.90 & 147.32 & 243.72 \\
\hline 25.79 & 132.61 & 132.63 & 132.85 & 133.71 & 147.14 & 243.62 \\
\hline 51.58 & 129.96 & 129.99 & 130.26 & 131.15 & 144.82 & 242.22 \\
\hline 77.37 & 128.06 & 128.68 & 128.73 & 129.66 & 140.02 & 237.04 \\
\hline 103.17 & 124.30 & 124.59 & 125.80 & 126.53 & 146.56 & 222.38 \\
\hline
\end{tabular}

Table 9

Second natural frequencies (in $\mathrm{Hz}$ ) clamped-clamped fluid-conveying pipe for various values of $\gamma_{1}$ and $V_{0}, \beta=0.6$

\begin{tabular}{|c|c|c|c|c|c|c|}
\hline \multirow{2}{*}{$V_{0}, \mathrm{~m} / \mathrm{s}$} & \multicolumn{6}{|c|}{$\gamma_{1}$} \\
\cline { 2 - 7 } & 0.01 & 0.5 & 2.5 & 10 & 100 & 1000 \\
\hline 0.00 & 362.98 & 363.01 & 363.12 & 363.53 & 367.65 & 415.46 \\
\hline 25.79 & 361.11 & 361.88 & 361.99 & 362.41 & 367.52 & 415.34 \\
\hline 51.58 & 360.11 & 360.14 & 360.25 & 360.67 & 365.80 & 413.82 \\
\hline 77.37 & 353.79 & 353.82 & 353.99 & 354.35 & 359.58 & 408.34 \\
\hline 103.17 & 337.12 & 337.14 & 337.27 & 337.72 & 343.21 & 394.03 \\
\hline
\end{tabular}




\section{Conclusions}

The present work is a contribution to the study of pipes vibration, hydrodynamic structures, through analytical and numerical approaches. A tool is used to intend the calculation of natural frequencies and visualize the flow in the pipe system filled on fluid. At first time, the hydrodynamic problem is treated by analyzing the first two natural modes of particular cases. Several examples were processed to determine the influence of the fluid velocity and different geometrical and physical parameters on the phenomenon of fluid-structure interaction. The main findings can be summarized as follows:

1. The first conclusion which one can draw from this study is that the frequencies of the system fluid-structure depend on the geometrical and physical properties.

2. The natural frequencies of the pipe conveying fluid depend on the velocity of the fluid. At a certain critical velocity the first natural frequency decreases until vanishes, the natural frequencies of this case can be obtained analytically.

3 . The increase in the $\beta$ (mass ratios) increased the value of the added mass to the system and therefore decreases the frequency parameter.

4. The attempt was made to validate the current formulation of the problem.

5 . The natural frequencies increase significantly with the values of $\gamma_{1}$, and increases sharply for $\gamma_{1}$ at around $10^{3}$.

6. The results with Winkler foundation parameter of a pinned-pinned pipe under flow are obtained analytically.

7. The dimensionless critical velocity of $\gamma_{1}$ values of pinned-pinned pipe is presented.

8 . To validate the numerical results, we carried out the analytical calculation of turbulent flow with $\beta=0.6$.

9. The results obtained numerically are similar to those obtained by the analytical approach for the determination of the natural frequencies. A global error on the first frequency of the order $1.15 \%$ is very satisfactory.

10. After the numerical validation, we can study the one-way fluid solid interaction of pipe under flow with different different physical and geometrical parameters.

11. Numerical simulation with ANSYS is therefore placed as a promising tool for the design of pipes, on the condition of being of a cost of accessible calculation. To obtain a very good precision, using mesh with hexahedral elements (SOLID 45). This code also makes it possible to determine all the modes of vibration of the pipe.

\section{References}

1. George, A. Antaki 2006. Piping and Pipeline Engineering: Design, Construction, Maintenance. Integrity, and Repair. Aiken, South Carolina, U.S.A., 564 p.

2. De Jong, C.A.F. 1994. Analysis of pulsations and vibrations in fluid-filled pipe systems, $\mathrm{PhD}$ Thesis, Eindhoven University of Technology.

3. Païdoussis, M.P; Li, G.X. 1993. Pipes conveying fluid: a model dynamical problema, Journal of Fluids and Structures 8: 137-204. http://dx.doi.org/10.1006/jfls.1993.1011.

4. Païdoussis, M.P. 1998. Fluid-Structure Interactions: Slender Structures and Axial Flow, vol. 1, Academic
Press, San Diego.

http://dx.doi.org/10.1016/S1874-5652(98)80001.

5. Anklin, M.; Drahm, W.; Gieder, A. 2006. Coriolis mass flow meters: overview of the current state of the art and latest research, Flow Measurement and Instrumentation 17: 317-323.

http://dx.doi.org/10.1016/j.flowmeasinst.2006.07.004.

6. Jon Juel Thomsen; Jonas Dahl 2010. Analytical predictions for vibration phase shifts along fluid-conveying pipes due to Coriolis forces and imperfections, Journal of Sound and Vibration 329: 3065-3081.

http://dx.doi.org/10.1016/j.jsv.2010.02.010.

7. Olson, L.G; Jamison, D. 1997. Application of a general purpose finite element method to elastic pipes conveying fluid, Journal of Fluids and Structures 11: 207-222.

http://dx.doi.org/10.1006/jfls.1996.0073.

8. Morteza, H. Sadeghi; Mohammad, H. Karimi-Dona 2011. Dynamic behavior of a fluid conveying pipe subjected to a moving sprung mass-An FEM-state space approach, International Journal of Pressure Vessels and Piping 88: 123-131.

http://dx.doi.org/10.1016/j.ijpvp.2011.02.004.

9. Lee, U.; Park, J. 2006. Spectral element modelling and analysis of a pipeline, Journal of Fluids and Structures 22: 273-292.

http://dx.doi.org/10.1016/j.jfluidstructs.2005.09.003.

10. Dai, H.L.; Wang, L.; Qian, Q.; Gan, J. 2012. Vibration analysis of three-dimensional pipes conveying fluid with consideration of steady combined force by transfer matrix method, Applied Mathematics and Computation 219: 2453-2464.

http://dx.doi.org/10.1016/j.amc.2012.08.081.

11. Ni, Q.; Zhang, Z.L.; Wang, L. 2011. Application of the differential transformation method to vibration analysis of pipes conveying fluid, Applied Mathematics and Computation 217: 7028-7038.

http://dx.doi.org/10.1016/j.amc.2011.01.116.

12. Jijun Gu, Chen An, Menglan Duan, Carlos Levi, Jian Su. 2013. Integral transform solutions of dynamic response of a clamped-clamped pipe conveying fluid, $\mathrm{Nu}-$ clear Engineering and Design 254: 237-245. http://dx.doi.org/10.1016/j.nucengdes.2012.09.018.

13. Xinwei Wang, Yongliang Wang 2013. Free vibration analysis of multiple-stepped beams by the differential quadrature element method, Applied Mathematics and Computation 219: 5802-5810.

http://dx.doi.org/10.1016/j.amc.2012.12.037.

14. Wang, Lin.; Qiao, Ni. 2008. Nonlinear dynamics of a fluid-conveying curved pipe subjected to motion-limiting constraints and a harmonic excitation, Journal of Fluids and Structures 24: 96-110. http://dx.doi.org/10.1016/j.jfluidstructs.2007.07.002.

15. Öz, H.R. 2001. Non-linear vibrations and stability analysis of tensioned pipes conveying fluid with variable velocity, International Journal of Non-linear Mechanics 36: 1031-1039. http://dx.doi.org/10.1016/S0020-7462(00)00065-2.

16. Chellapilla, K.R.; Simha, H.S. 2007. Critical velocity of fluid-conveying pipes resting on two-parameter foundation, Journal of Sound and Vibration 302: 387-397. http://dx.doi.org/10.1016/j.jsv.2006.11.007.

17. Chellapilla, K.R.; Simha, H.S. 2008. Vibrations of fluid-conveying pipes resting on two-parameter foundation, The Open Acoustics Journal 1: 24-33. 
http://dx.doi.org/10.1016/j.jsv.2006.11.007.

18. Makerle, J. 2000. Finite element vibration and dynamic response analysis of engineering structures, Shock Vibration 7: 39-56. http://dx.doi.org/10.1155/2000/405046.

19. Olson, L.G.; Amison, D.J. 1997. Application of a general purpose finite element method to elastic pipes conveying fluid, Journal of Fluids and Structures 11: 207222. http://dx.doi.org/10.1006/jfls.1996.0073.

20. Zou, G.P.; Cheraghi, N.; Taheri, F. 2005. Fluid-induced vibration of composite natural gas pipelines, International Journal of Solids and Structures 42: 1253-1268. http://dx.doi.org/10.1016/j.ijsolstr.2004.07.001.

21. Pierre-Antoine Emorine, Alexandre Veillon, Jerome Vinconneau 2010. Modélisation des interactions fluidestructure, LAMIH ENSIAME C2S 2a Rapport de Projet.

22. Singiresu, S. Rao 2011. Mechanical Vibrations 5th ed, Pearson Education, Inc., publishing as Prentice Hall, 1 Lake Street, Upper Saddle River, NJ 07458.

23. Païdoussis, M.P. 2005. Some unresolved issues in fluidstructure interactions, Journal of Fluids and Structures 20: 871-890.

http://dx.doi.org/10.1016/j.jfluidstructs.2005.03.009.

24. Schmucker, H.; Flemming, F.; Coulson, S. 2010. Two-way coupled fluid structure interaction simulation of a propeller turbine, IOP Conference Series: Earth and Environmental Science 12(1): 012011. http://iopscience.iop.org/article/10.1088/1755-1315/12/ $1 / 012011$.
M. Dahmane, D. Boutchicha, L. Adjlout

\section{ONE-WAY FLUID STRUCTURE INTERACTION OF PIPE UNDER FLOW WITH DIFFERENT BOUNDARY CONDITIONS}

\author{
S u m m a r y
}

This paper deals with the analytical modelisation and the numerical simulation of fluid structure interaction (FSI) phenomena of pipeline. The equations of motions and equilibrium are coupled together taking into account the influence of the fluid flow inside the pipe. The fluid circulating in the pipe has the flexional motion as that of structure. The analytical model is based on the Newtonian approach. The practicability of the calculation model and the effects of fluid-structure interaction are illustrated by calculations for some simple systems, for pipe with boundary condition pinned-pinned. Independently of the analytical method, a numerical modal analysis is realized in the fluid structure configuration. This configuration has been simulated by coupling the two commercial solvers: Fluent code of fluid mechanical (CFD, Computational Fluid Dynamics), and ANSYS classical code of structure mechanical (CSD, Computational structure Dynamics) for pipes with different boundary conditions: pinned-pinned, clamped-pinned and clamped-clamped. We describe the technique used to solve the equations associated in the determination of natural frequencies. For this, we use code ANSYS Workbench where the equations are discritized with finite element method. The results obtained numerically are similar to those obtained by the analytical approach.

Keywords: Fluid conveying pipes frequency, fluid structure interaction, Galerkin, foundation, finite element.

Received September 20, 2015 Accepted November 25, 2016 